Improved Safety Valves. By Alfred Gregory, EsQ., C. E.

From the London Mechanics' Magazine, for November, 1848.

I have invented an improved form of safety valve for steam boilers, which has met with the approbation of several eminent engineers. I am desirous of giving it publicity, and for that purpose respectfully submit the accompanying sketches and description, for insertion in an early number of the Mechanics' Magazine.

The advantages do not require to be much insisted on. The practice of overloading the safety valve is much more common than is generally supposed: sometimes it is the act of ignorance, but most frequently that of will, not only endangering life, but injuring the pocket also, in the destruction (which is very serious) that ensues to the boilers, fire-boxes, \&c. The number of fines levied on locomotive engine-men furnishes sufficient evidence of the frequency of its occurrence.

Fig. 1.

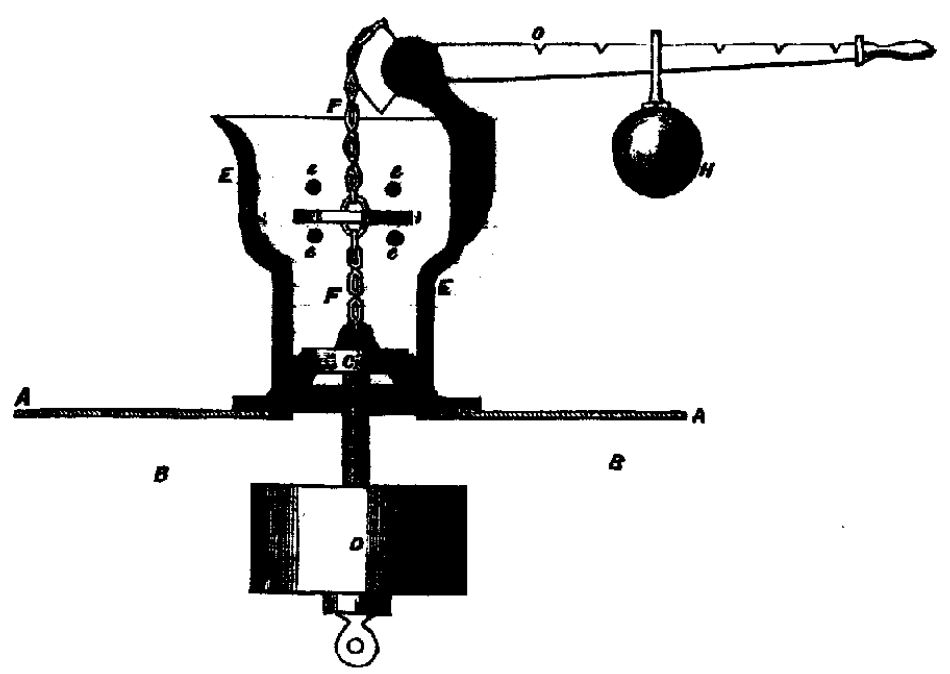

\title{
Description.
}

Fig. 1, represents the safety valve in a form appropriate for stationary use.

Fig. 2, another modification, on the same principle, applicable to boilers of every description, locomotive, marine, and stationary.

Fig. 1, AA, represent the top plates of boiler; BB, the steam space inside; $\mathrm{C}$, the ordinary conical valve, having a perpendicular spindle, to which a weight, $\mathrm{D}$, is attached; $\mathrm{EE}$, valve-box; FF, a chain, which is connected to the valve at one end, and at its other end to the short curved part of the lever, $\mathrm{H}$, is a regulating weight; $\mathrm{I}$, a shield to protect the valve from injury or interference; $e, e, e, e$, four bars or stops to the shield I, for frustrating any attempt to damage the valve.

The mode of working is, for the steam to raise the valve, as usual, when 
its pressure excceds that of the weight, $D$, less the lifting power of the lever, $G$, and the weight, $H$. The weight, $D$, being inside the boiler, cannot be for any mischievous purpose got at; and it is equal to the extreme pressure allowed upon the boiler, or may be made so, by hanging weights to the eye-loop of $\mathrm{D}$, which additional weights may be reduced, or altogether removed, as the boiler deteriorates by wear. For any less pressure, according to the working necessities of the engine, the engineer has the same control over the valve as at present, by sliding the weight, $\mathrm{H}$, on the lever, $\mathrm{G}$, which operates in taking off weight; regulating the reduction as he pleases; but he cannot increase the load upon the valve beyond what the weight, $D$, inside the boiler, gives; for, if hanging more weight on the lever, $G$, he takes of instead of increasing pressure; and, on the other hand, if raising the handle end of the lever, it has no effect upon the valve on account of the connecting medium being $a$ chain, which, of course, can only operate in one way, hanging loose, as it does, and throwing no stress upon the valve when moving downwards.

Fig. 2.

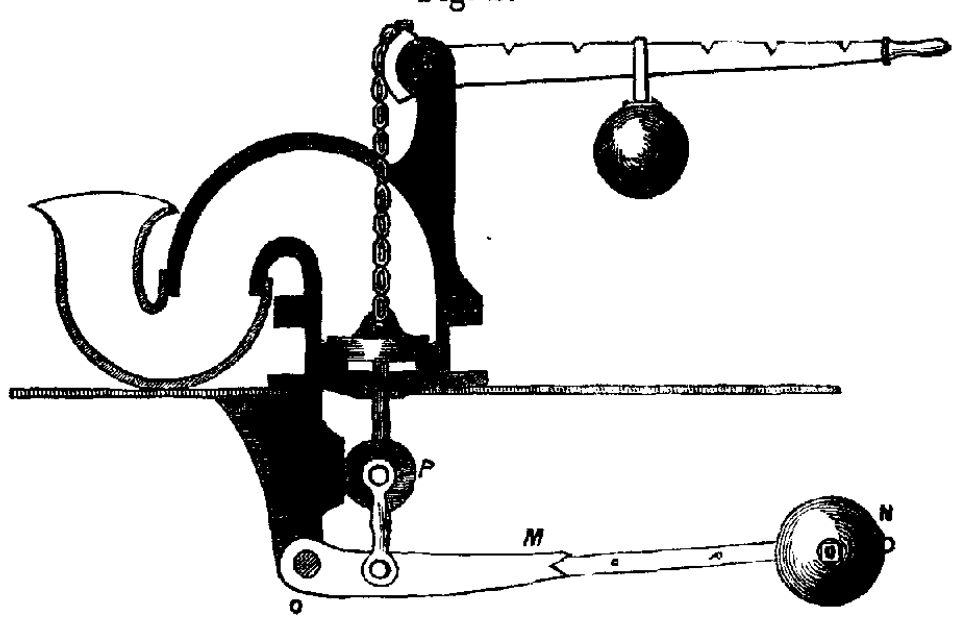

Fig. 2 is another form, involving the same action, but instead of the heavy weight altached to the valve-spindle, as before, there is, inside the boiler, a lever, $M$, having a weight, $N$, and fulcrum at $O$, equal in its effect to the extreme pressure allowed, and which may be reduced as the boiler, by use, weakcns, by fixing the weight, $\mathrm{N}$, nearer the fulcrum, convenience for which is shown. Instead, also, of the protecting shield, as in No. 1, the blow-away steam is here carried off by a double U-bent pipe, the accessible half of which is made of thin sheet copper, strong enough to carry away the stean as it blows freely into the air, there being then little or no pressure; but if the steam be confined within it, through any wilful attempt to plug the pipe up, the copper will rend, (a result peculiar to that metal,) and permit the necessary escape. There is likewise shown a small roller, $\mathbf{P}$, to counteract the curvilineal action of the lever $M$, and keep the valve-spindle from "sticking." But both this and the copper 
pipe are precautions no more necessary than at present with the best constructed valves, and might safely be dispensed with. The remaining parts and action of figure 2 are the same as figure 1.

Both plans represent the principle of a valve, the mechanical arrangement of which, however modified, embraces every advantage as to security, of a "locked valve," in frustrating any attempt to overload it, either by accident or design, through ignorance or will, without the usual attendant disadvantages of inconvenience, expense, \&c. of a second valve, and liability to "stick" by corrosion of parts through standing long unused, \&c.; a liability which the present form has not, as it is the engineers' frequent "working" safety valve, which is a locked and limited one, but possessing all the facility of regulating his pressure that he has now, though not allow ed to exceed the fixed extreme safe point.

\section{On the Duration of a Solar Spot.}

BY W. PRINGLE, ESQ.

It is stated by Dr. Dick and other writers that "no spot has been known to last longer than one that appeared in the year 1676 , which continued upon the sun for seventy days." I am inclined to think that a large spot now going off the sun's disk (Nov, 21), bas lasted still longer.

On the 19th of August last I observed a double spot, or two adjacent spots, near the sun's eastern verge, which as they advanced increased in size, and on the 23rd were visible to the naked eye as one spot. Their longest diameters were 27,000 and 31,000 miles respectively, being each about a third less in breadth, embracing the penumbræ. They were about 10,000 miles apart, though for the first two or three days they appeared to be linked together by a curved chain of minute spots or shallows at their northern extremities, but which became eventually absorbed into the penumbræ of the largest of the two. They were both of an oval form, and were followed till the 28th or 29th, when the nucleus of one had divided into several parts. They probably disappeared on the 31st of August.

On September 16, I remarked a large spot (at 4 P. M.) so far advanced on the sun's eastern limb as distinctly to exhibit several black nuclei within the edges of the penumbræ, but no great nucleus in the centre; and on the

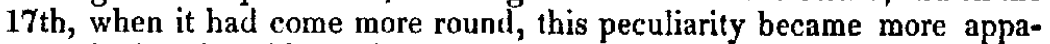
rent, the interior sides being studded with small dark spots, while the centre showed nothing but one vast clouded space or shallow. As I had been looking for the re-appearance of the two spots of August, it occurred to me that they might have become conjoined, the conjunction of the two penumbrax forming the central space of the spot now advancing. 'There being no other traces of them, I conceived I was justified in this conclusion. The smooth contour of the two oval spots had become since the contact a vast irregular polygon, as if by the concussion of two forces the penumbræ had been dashed or shaken into angular protrusions. The entire spot on the 17th measured about 50,000 miles in its longest dimensions. A very fair engraving of its outline appeared in the Illustrated London News of October 7 th. Its size on the 21st was estimated at 60,000 miles: I made it somewhat more afterwards. It was quite percep- 